\title{
Molecular dynamics simulations and photoluminescence measurements of annealed $\mathrm{ZnO}$ surfaces
}

\author{
Tjun Kit Min ${ }^{\mathrm{a}}$, Tiem Leong Yoon ${ }^{\mathrm{a}, *}$, Chuo Ann Ling ${ }^{\mathrm{a}}$, Shahrom Mahmud ${ }^{\mathrm{a}}$, \\ Thong Leng Lim ${ }^{c}$, Kim Guan Saw ${ }^{\mathrm{a}, \mathrm{b}}$ \\ ${ }^{a}$ School of Physics, Universiti Sains Malaysia, 11800 USM, Penang, Malaysia \\ ${ }^{b}$ School of Distance Education, Universiti Sains Malaysia, 11800 USM, Penang, Malaysia \\ ${ }^{c}$ Faculty of Engineering and Technology, Multimedia University, Jalan Ayer Keroh \\ Lama, 75450 Melaka, Malaysia
}

\begin{abstract}
The effect of thermal annealing on wurtzite $\mathrm{ZnO}$, terminated by two surfaces, $\left(\begin{array}{llll}0 & 0 & 0 & \overline{1}\end{array}\right)$ (which is oxygen-terminated) and $\left(\begin{array}{llll}0 & 0 & 0 & 1\end{array}\right)$ (which is Znterminated), is investigated via molecular dynamics simulation using reactive force field (ReaxFF). As a result of annealing at a threshold temperature range of $700 \mathrm{~K}<T_{\mathrm{t}} \leq 800 \mathrm{~K}$, surface oxygen atoms begin to sublimate from the $\left(\begin{array}{llll}0 & 0 & 0 & \overline{1}\end{array}\right)$ surface, while no atom leaves the $\left(\begin{array}{llll}0 & 0 & 0 & 1\end{array}\right)$ surface. The ratio of oxygen leaving the surface increases with temperature $T$ (for $T \geq T_{\mathrm{t}}$ ). The relative luminescence intensity of the secondary peak in the photoluminescence (PL) spectra, interpreted as a measurement of amount of vacancies on the sample surfaces, qualitatively agrees with the threshold behavior as found in the MD simulations. Our simulations have also revealed the formation of oxygen dimers on the surface and evolution of partial charge distribution during the annealing process. Our MD simulation based on the ReaxFF is consistent with experimental observations.
\end{abstract}

Keywords:

Molecular Dynamics; Reactive Force Field; Annealing; ZnO surface

${ }^{*}$ Corresponding author

Email addresses: klorin2002@yahoo.co.uk (Tjun Kit Min ), tlyoon@usm.my (Tiem Leong Yoon ), lingchuoann@hotmail.com (Chuo Ann Ling), shahromx@usm.my (Shahrom Mahmud), tllim@mmu.edu.my (Thong Leng Lim), kgsaw@usm.my (Kim Guan Saw) 


\section{Introduction}

ZnO has been extensively studied, both theoretically and experimentally, due to its many promising applications in piezoelectric devices, transistors, photodiodes, photocatalysis and antibacterial function [1, 2, 3]. The physical properties of $\mathrm{ZnO}$, especially its surface properties, can be experimentally modified at the atomic level for the purpose of, e.g., engineering the material for desired functionality. Since $\mathrm{ZnO}$ contacts with its external environment through its surfaces, monitoring how the surface properties respond to external perturbation (e.g. thermal treatment) can provide valuable insights to improve our ability to manipulate $\mathrm{ZnO}$ for application purposes. One of the simplest way to modify the surfaces of $\mathrm{ZnO}$ is by heating it to high temperatures (below its melting point). Heating $\mathrm{ZnO}$ can be easily carried out in practice, and many works had been reported along this line [4, 5, 6].

$\mathrm{ZnO}$ crystals are dominated by four surfaces with low Miller indices: the non-polar $\left(\begin{array}{llll}1 & 0 & \overline{1} & 0\end{array}\right)$ and $\left(\begin{array}{llll}1 & 1 & \overline{2} & 0\end{array}\right)$ surfaces and the polar surfaces which are the zinc-terminated surface $\left(\begin{array}{llll}0 & 0 & 0 & 1\end{array}\right)$ and the oxygen terminated surface $\left(\begin{array}{ll}0 \\ 0\end{array}\right.$ $\left.\begin{array}{lll}0 & 0 & \overline{1}\end{array}\right)$. Surface energy of polar surfaces in an ionic model diverges with sample size due to the generation of a macroscopic electrostatic field across the crystal [7]. This kind of behavior was well investigated by Tasker [8]. This is why wurzite $\mathrm{ZnO}$ is labeled as Tusker-type surfaces, which are formed by alternating layers of oppositely charged ions.

In this work we investigate what will happen to the atomic configuration of the surface when a $\mathrm{ZnO}$ slab with finite thickness is heated without melting. Sublimation of atoms from the polar surfaces due to temperature effect was studied using molecular dynamics (MD) simulation, where the trajectories of all atoms at a given temperature $T$ are followed quantitatively. In the MD simulated annealing, in which the reactive force field (ReaxFF) for $\mathrm{ZnO}$ is used, sublimation of $\mathrm{O}$ atoms from $\mathrm{ZnO}$ polar surface is observed. ReaxFF for $\mathrm{ZnO}$ allows for bond formation and charge transfer among the selected atoms. When the sublimation of atoms occurs, point vacancies are created on the surface. Quantitative information of the amount and type of atoms sublimated, as well as point vacancies created on the surface at different annealing temperatures can thus be obtained.

Experimentally, if we heat a $\mathrm{ZnO}$ wurtzite surface to an elevated temperature and investigate the resultant surface using photoluminescence (PL) measurement, the spectrum should reflect the amount of point vacancies

created. We expect that an increase of annealing temperature will create 
more point vacancies. In this paper, the predictions of MD simulation using ReaxFF are compared with the PL data.

In Section 2 we describe the MD procedures used to perform the simulations. In Section 3 we describe the experimental procedure to perform PL measurement on the $\mathrm{ZnO}$ samples. In Section 4, the experimental data and simulation results are discussed, compared and interpreted. The conclusion is in Section 5 .

\section{Molecular dynamics simulation procedure}

Crystalline $\mathrm{ZnO}$ can exist in various polymorphs. The stability of the polymorphs is dependant upon the pressure and temperature [9]. These polymorphs include wurtzite, zinc blende and rocksalt. $\mathrm{ZnO}$ in wurtzite structure is the most stable form at room pressure. The parameters of crystal structure of the wurtzite $\mathrm{ZnO}$ unit cell were shown in Table 1. A slab comprising $15 \times 15 \times 3$ unit cells was constructed. The slab was sandwiched between two vacuum layers of thickness $100 \AA$, while the thickness of the slab itself was $15 \AA$. The surface area of the slab in the supercell was $1783 \AA^{2}$. The thickness of vacuum was chosen such that the interaction between adjacent surfaces of two neighbouring supercells were negligible so as not give rise to any significant effect on the simulation outcome. Periodic boundary condition was imposed in all $x$-, $y$ - and $z$-directions. Fig. 1 shows the supercell of the ZnO slab. In all simulations the total number of atoms in the simulation box were maintained at 2700. The MD simulation throughout this work was carried out using the MD code LAMMPS [10].

The stepsize throughout the simulation was $\Delta t=0.5$ fs. The structure was first optimized at $0.1 \mathrm{~K}$ using the built-in conjugate gradient minimizer available in the LAMMPS package. We set the convergence criterion to be 10000 steps (generally, convergence is achieved in less than 100 steps). The temperature of the system was then heated up to $T_{\mathrm{r}}=300 \mathrm{~K}$ in 5000 steps. The system was further equilibrated for 20000 steps at $300 \mathrm{~K}$. At the end of the equilibration at $T_{\mathrm{r}}$, the temperature was raised to a chosen target temperature, $T$, at a rate of $5 \times 10^{10} \mathrm{~K} / \mathrm{s}$. The evolution of the system at annealing temperature was then followed at constant $T$ for a total of $N_{\text {step }}$ $=250000$ steps. The temperature of the system was then quenched from $T$ to $0.1 \mathrm{~K}$ at a rate of $5 \times 10^{10} \mathrm{~K} / \mathrm{s}$. A typical temperature vs. step profile is shown in Fig. 2 for annealing temperature $T=1000 \mathrm{~K}$. Nose-Hoover thermostat (NVT) was used throughout the simulation to control the temperature. The 
damping constant for the thermostat was set to 5 fs. The total steps in each simulation are dependent upon the target temperature $T$. The total duration for the simulations range from 315000 steps (0.16 ns) to 387000 steps (0.20 ns).

Independent simulations were also carried out for each target temperature $T$, ranging from $300 \mathrm{~K}$ to $1300 \mathrm{~K}$ at an interval of $100 \mathrm{~K}$. Atoms from the polar surfaces will leave the surface if the target temperature is sufficiently high. In this case we have to assure that $N_{\text {step }}$ that defines the length of the temperature plateau is sufficiently long such that atom will stop leaving the surface even if we continue to equilibrate at that annealing temperature. This precaution is necessary so that the conclusion of the MD outcome remains the same should a larger $N_{\text {step }}$ is used instead. After some trial-and-error efforts, it was found that a value of $N_{\text {step }}=250000$ steps was optimal for the range of temperatures investigated in our work. At the end of the simulation, i.e. when the slab has been quenched to $0.1 \mathrm{~K}$ from a given target temperature $T$, the type and total number of atoms leaving the surface were quantified. After running a series of simulation for various $T$, we would have collected sufficient data to plot the ratio of atoms leaving the surface as a function of annealing temperature.

The reliability of the MD results depends crucially on the force field used. Force field based on the ReaxFF model, first proposed in [11], allows bond formation and charge transfer to occur among the interacting atoms in the simulation box. In this work, the ReaxFF for $\mathrm{ZnO}$ is adopted. The $\mathrm{ZnO}$ reactive force field has been applied to the calculation of atomic vibrational mean square amplitudes for bulk wurtzite-ZnO for a temperature up to $600 \mathrm{~K}$ and found good agreement with experimental observations [12]. It has also been applied to study surface growth mechanism for the $\left(\begin{array}{llll}0 & 0 & 0 & 1\end{array}\right)$ surface and water molecule adsorption on stepped $\mathrm{ZnO}$ surfaces [13. The parameters of the ReaxFF used in this work was the same as that used in [13].

Through ReaxFF the process of equilibration of partial charge distribution among the atoms can be revealed in the MD simulation. In our simulations, all the atoms in the simulation box were assigned a partial charge of zero initially. While total charge of the system remains zero, partial charges among the atoms will redistribute itself as thermal equilibration process progresses. We followed the time evolution of partial charge distribution in the slab when the $\mathrm{ZnO}$ is going through the annealing process. The distributions of the partial charge density in the slab at the end of a MD run were obtained by post-processing the MD data. 


\section{Experimental details of photoluminescence measurements}

Photoluminescence (PL) measurements were carried out to justify the defect caused by the annealing procedure. Two sets of $\mathrm{ZnO}$ powder were used as starting material in this study. They were synthesized using French process [14], having different major morphological particles: rod (ZnO-1) and plate structures $(\mathrm{ZnO}-2)$. The $\mathrm{ZnO}$ powder were converted into pellets form using the $\mathrm{ZnO}$ agglomeration method, which was introduced in the previous work [14. The $\mathrm{ZnO}$ pellets formed using this technique retained the morphological structure of ZnO. X-ray diffraction (XRD) analysis had been carried out on the $\mathrm{ZnO}$ pellets and revealed the wurtzite polycrystalline structure of the $\mathrm{ZnO}$ samples (not shown here).

The $\mathrm{ZnO}$ pellets were subsequently annealed in nitrogen rich ambient for an hour with the gas flow regulated at $2.4 \mathrm{~L} / \mathrm{min}$. The annealing process was done in temperature manipulated at $573 \mathrm{~K}-1073 \mathrm{~K}$. The PL measurements were taken using the Jobin Yvon HR 800UV system with the HeCd Laser 325 $\mathrm{nm}$ as excitation source. The level of defect formation was also determined from the luminescence intensity.

\section{Results and Discussion}

\subsection{Sublimation of $O$ atoms at and beyond a threshold temperature $T_{t}$}

We have simulated the annealing of the wurtzite $\mathrm{ZnO}$ slab from $T=300 \mathrm{~K}$ to $T=1300 \mathrm{~K}$ at a $100 \mathrm{~K}$ interval. At low temperatures, the atoms are observed to execute relatively mild thermal vibration about their equilibrated positions. At higher temperatures, the vibration become more violent and the configuration of the atoms on both surfaces became more distorted as compared to the low temperature cases. However, the layered structure formed by the alternating layers of oxygen and $\mathrm{Zn}$ atoms remains visibly recognizable.

The video of the simulations were visually monitored for any atoms leaving the surfaces of the $\mathrm{ZnO}$ slab. We observed that $\mathrm{O}$ atoms began to leave the $\left(\begin{array}{llll}0 & 0 & 0 & \overline{1}\end{array}\right)$ surface, mostly in pairs, for simulation running with $T=800 \mathrm{~K}$ and above. Sublimation of the $\mathrm{O}$ atoms occurs only during the temperature plateau phase. No atoms other than the $\mathrm{O}$ atoms on the $\left(\begin{array}{llll}0 & 0 & 0 & \overline{1}\end{array}\right)$ surface sublimates from the slab. In particular, no Zn atom sublimates from either surfaces of $\mathrm{ZnO}$. Such observation tallies with the experimental measurement that only oxygen vacancies were created but not Zn upon heating a $\mathrm{ZnO}$ sample (see the related discussion in subsection 4.4). 


\subsection{Ratio of surface $O$ atoms sublimated as a function of annealing temper- ature}

Fig. 3 shows the ratio of $\mathrm{O}$ atoms sublimated to the total $\mathrm{O}$ atoms originally lying on the $\left(\begin{array}{llll}0 & 0 & 0 & \overline{1}\end{array}\right)$ surface for each target temperature. No sublimation occurs for $T=300,400, \cdots 700 \mathrm{~K}$. O atoms sublimate from its polar surface only happened for $T=800 \mathrm{~K}$ or above, with even more atoms leaving the $\left(\begin{array}{llll}0 & 0 & 0 & \overline{1}\end{array}\right)$ surface at larger $T$. However, Fig. 3 seems to suggest the possible existence of a threshold temperature, $700 \mathrm{~K}<T_{\mathrm{t}} \leq 800 \mathrm{~K}$, at which sublimation of $\mathrm{O}$ atoms from the $\mathrm{ZnO}$ surface begins to occur. We shall qualitatively compare this prediction against experimentally measured data in subsection 4.4 .

\subsection{Sublimation of surface $O$ atoms in pairs}

It was also observed that most of the sublimated $\mathrm{O}$ atoms will leave the $\left(\begin{array}{llll}0 & 0 & 0 & \overline{1}\end{array}\right)$ surface in pairs. A closer investigation of the simulated snapshots revealed that the two oxygen atoms forming a pair during sublimation were neighbors to each other before both left the surface. However we also observed a tiny portion of sublimated $\mathrm{O}$ atoms leaving the surface alone and did not form pairs.

The formation of oxygen atom pairs during sublimation was made possible in the MD simulation due to the novel capability of the reactive force field to allow bond formation between neighboring $\mathrm{O}$ atoms. From theoretical point of view, surface atoms are under very different conditions than that for the bulk atoms, give rise to the presence of dangling bonds in the former. As a result, the polar surface which consists of $\mathrm{O}$ atoms have higher tendency to form bond with a neighboring $\mathrm{O}$ atoms from the same surface layer. When sublimated, these dimers form oxygen pairs as observed in the MD simulation. Based on our MD results, we conclude the following microscopic picture: Dimers of $\mathrm{O}$ atoms will be formed on the wurtzite $\left(\begin{array}{llll}0 & 0 & 0 & \overline{1}\end{array}\right)$ $\mathrm{ZnO}$ surface when it is heated up. These dimers shall leave the surface in pairs if subsequently sublimated.

\subsection{Comparison with results from experiment measurements}

The experimental data obtained from PL measurement as described in Section 3 is shown in Fig. 4. The origin of the primary peak (at wavelength $382 \mathrm{~nm}$ ) in the PL data is due to the bandgap edge as well as excitonic recombination. The origin of the broad PL secondary peak, i.e. green emission centered at $536 \mathrm{~nm}$, remains a debatable issue. It could be interpreted as 
a reliable indicator of the abundance of point defect present on the surface or otherwise. In either case, it is reasonable to attribute the intensity of the green emission to the presence of point defects. The only issue is the quantitative extent of the contribution of the point defect apart from other possible causes. In this paper we adopt the viewpoint that the intensity of the secondary PL peak is taken as a qualitative indicator of the abundance of point defect present on the surface. Such a viewpoint is consistent with the other reports [15, 16, 17, 18, 19. Ahn et al. had explained that the broad green peak centered at $536 \mathrm{~nm}$ is due to band transition from zinc interstitial $\left(\mathrm{Zn}_{i}\right)$ to oxygen vacancy ( $\left.\mathrm{Vo}\right)$ defect levels in $\mathrm{ZnO}$ [15]. Alvi et. al. reported the band transition from $\mathrm{Zn}_{i}$ to $\mathrm{Vo}$ level is approximately $2.31 \mathrm{eV}$ (green emission) based on the full potential linear muffin-tin orbital method [16].

We will interpret the PL data by making the following assumptions:

1. The larger the abundance of oxygen point defects on the surface the higher the intensity of the secondary peak;

2. The primary peak contribution to the oxygen vacancies on the surface is not to be considered in present analysis (which is still qualitative) 1

Fig. 5 shows the intensities of green luminescence (GL) at wavelength $512 \mathrm{~nm}$ of the $\mathrm{ZnO}-1$ and $\mathrm{ZnO}-2$ samples as a function of temperature. The $\mathrm{ZnO}$ samples at $T=300 \mathrm{~K}, T=573 \mathrm{~K}, 673 \mathrm{~K}, 773 \mathrm{~K}$ and $873 \mathrm{~K}$ display a low intensity. There is a pronounced increase of GL luminescence at $T=$ $973 \mathrm{~K}$ and $T=1073 \mathrm{~K}$, with the intensity at the latter temperature higher than the former. When $T$ crosses $873 \mathrm{~K}$ to $973 \mathrm{~K}$, both $\mathrm{ZnO}-1$ and $\mathrm{ZnO}-2$ samples (with different morphologies) display the same threshold effect of abrupt increase in the GL intensity. A temperature threshold effect is clearly being triggered between $T=873 \mathrm{~K}$ and $T=973 \mathrm{~K}$, which is to be compared with $700 \mathrm{~K}<T_{\mathrm{t}} \leq 800 \mathrm{~K}$ predicted by the MD simulation.

The predicted threshold temperature is based on an idealized model in a MD simulation that cannot exactly describe what actually happened in a real experiment. Since the parametrisation of the $\mathrm{ZnO}$ ReaxFF does not include sublimation of $\mathrm{O}$ atoms from $\mathrm{ZnO}$ surfaces, the value of $T_{\mathrm{t}}$ from $\mathrm{MD}$ falls so closely to the experimentally measured range of $873 \mathrm{~K}-973 \mathrm{~K}$ is a very suggestive finding. If we accept an uncertainty of $\sim \pm 100 \mathrm{~K}$ in the threshold temperature, which is reasonably acceptable for a typical MD calculation,

\footnotetext{
${ }^{1}$ Detailed analysis and interpretation of the primary peaks in the PL spectrum for the $\mathrm{ZnO}$ samples measured in present study is to be discussed elsewhere.
} 
then the following picture seems appropriate (at least qualitatively): (i) Oxygen point defects on the wurtzite $\mathrm{ZnO}\left(\begin{array}{llll}0 & 0 & 0 & \overline{1}\end{array}\right)$ surface are created only at or above the threshold annealing temperature of $T_{\mathrm{t}}$, and $(i i)$ the abundance of oxygen point defects on the surface increases as the annealing temperature $T$ increases (where $T \geq T_{\mathrm{t}}$ ).

Apart from the PL data as reported above, the MD simulation results are also consistent with a previous experiment [20] which was conducted by two present authors (C.A.L and S.M). In 20, two different $\mathrm{ZnO}$ nanostructures (in wurtzite phase) were investigated using electron spectroscopy imaging (ESI). One sample was not annealed while the other was annealed to $T=700^{\circ} \mathrm{C}(=973 \mathrm{~K})$ under $N_{2}$-rich condition. The surfaces of the samples were scanned for $\mathrm{Zn}$ and $\mathrm{O}$ atoms. The abundance of these atoms on the surfaces could be visually inspected and were characterized by two distinct colors. The images of the ESI measurement on the white $\mathrm{ZnO}$ samples were reproduced in Fig. 6. It was found that the density of $\mathrm{Zn}$ atom count is similar for both annealed and unannealed samples. However, the density of the $\mathrm{O}$ atoms count for the annealed sample has clearly decreased compared to the unannealed sample. The ESI images are consistent with the notion that O atoms will be sublimated at a temperature $T \geq T_{\mathrm{t}}$, while $\mathrm{Zn}$ atoms will not. Although the $\mathrm{ZnO}$ samples were measured for only two temperatures (one at ambient and the other at $973 \mathrm{~K}$ ) in [20], the data is ubiquitously consistent with the present MD results.

The qualitative agreement between the MD results and the two experimental measurements discussed above is encouraging. It provides a motivation to capitalize the MD simulation as a reliable means to derive useful information of the detailed mechanism of the $\mathrm{ZnO}$ surface undergoing annealing at the atomistic level, which is otherwise difficult to obtain via experimental approach alone.

\subsection{Partial charge distribution}

The time evolution of the partial charge distribution among the atoms in the $\mathrm{ZnO}$ sample can be followed throughout a MD course (during which the slab is undergoing a temperature history as in Fig. 2). As an illustration, Figs. 7, 8 display the snapshots of the partial charge distribution in the slab at various steps during the MD simulation at target temperatures $T=300 \mathrm{~K}$ and $T=1300 \mathrm{~K}$, respectively. In these figures, the blobs centered around a given $z$-position (representing the vertical distance from the $\left(\begin{array}{llll}0 & 0 & 0 & \overline{1}\end{array}\right)$ surface $)$ are made up of discrete dots, each represents the partial charges of an atom 
at the depth $z$. Partial charge with a positive (negative) value is associated with Zn (oxygen) atom.

The partial charge distribution for the unannealed slab (i.e. (a) in Fig. 7 and Fig. 8) displays an oscillation pattern. The oscillation is due to the fact that the sign of partial charges from the same atom type at a common layer is opposite to that of adjacent layers occupied by the other atom type. In our MD simulations using ReaxFF model, the partial charge of $\mathrm{O}$ atom is negative, while it is positive for $\mathrm{Zn}$ atom. The signs are in accordance with what we expected from both atom types based on their known electronegativity. When probing into the depth of the slab along the $z$-direction beginning from the $\left(\begin{array}{llll}0 & 0 & 0 & \overline{1}\end{array}\right)$ surface termination, atomic layers with alternating partial charges will be passed through in succession, with the first encountered layer being the negatively charged $\mathrm{O}$ layer. Oscillation of the partial charge density in the slab observed here has a strong resemblance to what is known as Friedel oscillations ${ }^{2}$ which lead to surface charge polarization, a common feature of all surfaces.

By following the snapshots sequentially in time, we can quantitative visualize the information of how the positions and partial charges of the atoms redistribute themselves throughout a MD course. For example, at the beginning, the blobs of points in (a) in Figs. 7, 8 are very concentrated. From this, it could be inferred that $(i)$ all atoms were arranged in layers according to their respective atom type, and (ii) atoms in the same layer share an approximately same value of partial charges. Atoms in the slab annealed to $300 \mathrm{~K}$ vibrate only mildly, as can be inferred from the smaller size of the blobs in (b) and (c) in Fig. 7. At the end of the annealing process at $300 \mathrm{~K}$, the atomic configuration of the slab, as well as the partial charges of the atoms, basically did not alter much. Fig. 8 shows that annealing the slab at $1300 \mathrm{~K}$ causes the atoms to vibrate violently about their atomic positions at the temperature plateau (blobs in (b) and (c) are more dispersed than in the $300 \mathrm{~K}$ case). At the end of the annealing process, the atomic positions became much distorted. The partial charges of the same atom type in a given layer spread to a broader value (as can be inferred from a large vertical spread in the blobs). Comparing graphs (a) and (d) in Fig. 8, the distortion resulted from the annealing process at $T=1300 \mathrm{~K}$ is particularly prominent

\footnotetext{
${ }^{2}$ For a brief discussion of Friedel oscillations, see for example, Chapter 11.2 in the textbook by Kaxiras [21].
} 
near the $\left(\begin{array}{llll}0 & 0 & 0 & \overline{1}\end{array}\right)$ side of the surface, where a large spread in partial charges and locations of the $\mathrm{Zn}$ atoms are observed. In general we found that the ( $\left.\begin{array}{llll}0 & 0 & 0 & \overline{1}\end{array}\right)$ end of the slab crumpled at the end of the annealing process for $T$ equal or larger than $300 \mathrm{~K}$.

Fig. 9 displays the partial charge distributions at the end of an MD run for eight annealing temperatures ranging from $400 \mathrm{~K}$ to $1100 \mathrm{~K}$. These figures are basically the compilation of stage (d) in Figs. 7, 8 for eight different $T$ ranging from $400 \mathrm{~K}$ to $1100 \mathrm{~K}$. A qualitative change in the partial charge distribution pattern between $T=700 \mathrm{~K}$ and $T=800 \mathrm{~K}$ was observed. For $T \leq 700 \mathrm{~K}$, the partial charge point were grouped in relatively concentrated blobs, which were slightly flattened in the horizontal direction. Upon crossing the temperature at $800 \mathrm{~K}$, the points of partial charges in these blobs disperse to a much larger extent along the $z$-axis.

To illustrate the temperature-induced abrupt change of partial charge distribution from a slightly different perspective, the density of net partial charges at a depth of $z$ from the $\left(\begin{array}{llll}0 & 0 & 0 & \overline{1}\end{array}\right)$ surface $\rho(z)$, which was derived from the partial charge distribution vs. $z$ data, is shown in Fig. 10 for annealing temperatures $T=500,700,800,1000 \mathrm{~K}$. The density of net partial charges at a depth $z, \rho(z)$, is defined as the sum of all partial charges in a volume element of $\Delta z A_{s}$, where $A_{s}$ is the surface area of the slab. The variation of $\rho(z)$ at different depth for a few $T$ shown in Fig. 10 displays a qualitative change in the density of partial charge profile when $T$ crosses $700 \mathrm{~K}$ to $800 \mathrm{~K}$. The qualitative change of the partial charge distribution coincides with the onset of $\mathrm{O}$ atoms sublimation in this temperature range described in subsection 4.1 .

\subsection{Partial charge of the sublimated $O$ atoms}

We have also enumerated the average partial charge of the sublimated $\mathrm{O}$ atoms at each temperature (Fig. 11). When the sublimated atoms first appeared at $T=T_{\mathrm{t}}$, the average net charge per atom was $\sim-0.5 e$, and this value increased to $\sim-0.7 e$ at $T=1300 \mathrm{~K}$. The temperature dependence of the average partial charges of the sublimated $\mathrm{O}$ atoms appeared to flatten out for $T$ beyond $1100 \mathrm{~K}$. When the $\mathrm{O}$ atoms were sublimated from the surface, they carry away with them a net negative charge, leaving behind a substrate that has an overall net positive charge on the surface. 


\subsection{The Zn-terminated surfaces, $\left(\begin{array}{llll}0 & 0 & 0 & 1\end{array}\right)$}

The $\mathrm{ZnO}$ slab constructed for the MD simulation contains two surfaces, i.e., $\left(\begin{array}{llll}0 & 0 & 0 & 1\end{array}\right)$ and $\left(\begin{array}{llll}0 & 0 & 0 & \overline{1}\end{array}\right)$. For the Zn-terminated $\left(\begin{array}{llll}0 & 0 & 0 & 1\end{array}\right)$ surface, which is on the other side of the slab, we have found no robust sign of sublimation of any atom. The observation suggests that $\mathrm{O}$ atoms only sublimate from the $\left(\begin{array}{llll}0 & 0 & 0 & \overline{1}\end{array}\right)$ surface in wurtzite $\mathrm{ZnO}$ upon annealing, while not so from the surface terminated by Zn atom. Our MD simulation results suggest the possibility that the $\mathrm{Zn}$ atom on the surface termination presents a strong attractive force to any $\mathrm{O}$ atom attempting to escape the surface via thermal excitation. Whether the same can be said for other surfaces terminated with Zn- or oxygen-zinc hybrid atoms is an interesting question worthy of further investigation. However such hypothesis needs to be tested more rigorously by investigating the detailed behavior of the force of the $\mathrm{Zn}$ atom on any $\mathrm{O}$ atom attempting to leave the surface.

\section{Conclusion}

We have performed MD annealing simulations of a wurtzite $\mathrm{ZnO}$ slab with a thickness of $15 \AA$ and a surface area of $1783 \AA^{2}$. The simulations are performed using $\mathrm{ZnO}$ ReaxFF. There are two polar surfaces, namely $(0$ $\left.\begin{array}{lll}0 & 0 & 1\end{array}\right)$ and $\left(\begin{array}{llll}0 & 0 & 0 & \overline{1}\end{array}\right)$. The slab is thermally annealed from $0.1 \mathrm{~K}$ in stages up to a target temperature $T$, and then further equilibrated at the constant $T$ plateau for sufficiently large number of steps before the temperature is gradually quenched to $0.1 \mathrm{~K}$. The common temperature history experienced by the polar surfaces are shown in Fig. 2 .

We have run MD simulations for a range of target temperature from $T=300 \mathrm{~K}$ till $1300 \mathrm{~K}$ at a $100 \mathrm{~K}$ interval. Our MD results show that $\mathrm{O}$ atoms on the $\left(\begin{array}{llll}0 & 0 & 0 & \overline{1}\end{array}\right)$ surface are sublimated whenever $T \geq T_{\mathrm{t}}$, where $700 \mathrm{~K}<T_{\mathrm{t}} \leq$ $800 \mathrm{~K}$. Otherwise no atom sublimates from either surface. The ratio of $\mathrm{O}$ atoms sublimated to the total number of $\mathrm{O}$ atoms on the surface increases as $T\left(T \geq T_{\mathrm{t}}\right)$ increases. The existence of a threshold temperature $T_{\mathrm{t}}$ at which $\mathrm{O}$ atoms from the $\left(\begin{array}{llll}0 & 0 & 0 & \overline{1}\end{array}\right)$ surface begins to sublimate qualitatively agrees with our PL data (obtained experimentally) and a previous qualitative observation 20] based on ESI imaging. The MD simulation results also show that the sublimated $\mathrm{O}$ atoms do so in pairs. Due to the geometrically asymmetric condition, atoms at the surface are expected to form dimers. The $\mathrm{O}$ pairs seen leaving the surface in the MD sublimation originate from these dimers. We have also investigated the partial charge density of the slab as a function 
of depth from the surface, $z$, after each slab has gone through an annealing history as depicted in Fig. 2. The oscillatory form of the charge density of the slab resembles Friedel oscillations which are expected for the charge density near a surface. A qualitative change in the partial charge distribution pattern was observed while the annealing temperature crosses $T=700 \mathrm{~K}$ to $T=800 \mathrm{~K}$. Thermally-driven sublimation of atoms only occurs in the Oterminated $\left(\begin{array}{llll}0 & 0 & 0 & \overline{1}\end{array}\right)$ surface, whereas no sublimation of either atom type would occur in the $\left(\begin{array}{llll}0 & 0 & 0 & 1\end{array}\right)$ surface which is Zn-terminated, presumably due to a strong attractive force exerted by the Zn atoms at these surfaces.

\section{Acknowledgements}

T. L. Yoon wishes to acknowledge the support of (1) FRGS grant FASA 2/2013 by the Ministry of Higher Education of Malaysia (203/ PFIZIK/6711348), (2) USM RU Grant (1001/PSOSIAL/816210). We also acknowledge the financial support from an ERGS grant (203/PFIZIK/6730100) from the Malaysian Government.

\section{References}

[1] J. Goldberger, D. J. Sirbuly, M. Law, P. Yang, Zno nanowire transistors, The Journal of Physical Chemistry B 109 (2005) 9-14. PMID: 16850973.

[2] Z. L. Wang, J. Song, Piezoelectric nanogenerators based on zinc oxide nanowire arrays, Science 312 (2006) 242-246.

[3] J. Becker, K. R. Raghupathi, J. St. Pierre, D. Zhao, R. T. Koodali, Tuning of the crystallite and particle sizes of zno nanocrystalline materials in solvothermal synthesis and their photocatalytic activity for dye degradation, The Journal of Physical Chemistry C 115 (2011) 1384413850 .

[4] E. S. Shim, H. S. Kang, S. S. Pang, J. S. Kang, I. Yun, S. Y. Lee, Annealing effect on the structural and optical properties of zno thin film on inp, Materials Science and Engineering: B 102 (2003) 366 - 369. E-MRS 2002 Symposium E: Advanced Characterisation of Semiconductors.

[5] S. Wei, J. Lian, H. Wu, Annealing effect on the photoluminescence properties of zno nanorod array prepared by a pld-assistant wet chemical method, Materials Characterization 61 (2010) 1239 - 1244. 
[6] L. H. Quang, S. J. Chua, K. P. Loh, E. Fitzgerald, The effect of postannealing treatment on photoluminescence of zno nanorods prepared by hydrothermal synthesis, Journal of Crystal Growth 287 (2006) 157 161. Proceedings of the International Conference on Materials for Advanced Technologies (ICMAT 2005) Symposium $\{\mathrm{NZnO}\}$ and Related MaterialsProceedings of the International Conference on Materials for Advanced Technologies (ICMAT 2005) Symposium N.

[7] G. Kresse, O. Dulub, U. Diebold, Competing stabilization mechanism for the polar zno(0001)-zn surface, Phys. Rev. B 68 (2003) 245409.

[8] P. W. Tasker, The stability of ionic crystal surfaces, Journal of Physics C: Solid State Physics 12 (1979) 4977.

[9] H. Morkoc, U. Ozgur, Zinc Oxide, Wiley VCH Verlag GmbH, 2009.

[10] S. Plimpton, Fast parallel algorithms for short-range molecular dynamics, Journal of Computational Physics 117 (1995) 1 - 19.

[11] A. C. T. van Duin, S. Dasgupta, F. Lorant, W. A. Goddard, Reaxff: A reactive force field for hydrocarbons, J. Phys. Chem. A 105 (2001) 9396-9409.

[12] D. Raymand, A. C. van Duin, M. Baudin, K. Hermansson, A reactive force field (reaxff) for zinc oxide, Surface Science 602 (2008) 1020 1031.

[13] D. Raymand, A. C. van Duin, D. Spangberg, W. A. G. III, K. Hermansson, Water adsorption on stepped zno surfaces from md simulation, Surface Science 604 (2010) 741 - 752.

[14] S. Mahmud, One-dimensional growth of zinc oxide nanostructures from large micro-particles in a highly rapid synthesis, Journal of Alloys and Compounds 509 (2011) 4035-4040.

[15] C. H. Ahn, Y. Y. Kim, D. C. Kim, S. K. Mohanta, H. K. Cho, A comparative analysis of deep level emission in zno layers deposited by various methods, Journal of Applied Physics 105 (2009) 013502.

[16] N. H. Alvi, K. ul Hasan, O. Nur, M. Willander, The origin of the red emission in n-zno nanotubes/p-gan white light emitting diodes, Nanoscale Research Letters 6 (2011) 130. 
[17] A. B. Djurisic, Y. H. Leung, K. H. Tam, Y. F. Hsu, L. Ding, W. K. Ge, Y. C. Zhong, K. S. Wong, W. K. Chan, H. L. Tam, K. W. Cheah, W. M. Kwok, D. L. Phillips, Defect emissions in zno nanostructures, Nanotechnology 18 (2007) 095702.

[18] K. H. Tam, C. K. Cheung, Y. H. Leung, A. B. Djurisic, C. C. Ling, C. D. Beling, S. Fung, W. M. Kwok, W. K. Chan, D. L. Phillips, L. Ding, W. K. Ge, Defects in zno nanorods prepared by a hydrothermal method, The Journal of Physical Chemistry B 110 (2006) 20865-20871. PMID: 17048900 .

[19] B. Lin, Z. Fu, Y. Jia, Green luminescent center in undoped zinc oxide films deposited on silicon substrates, Applied Physics Letters 79 (2001) 943-945.

[20] L. C. Ann, S. Mahmud, S. K. M. Bakhori, Electron spectroscopy imaging and surface defect configuration of zinc oxide nanostructures under different annealing ambient, Applied Surface Science 265 (2013) 137 144.

[21] E. Kaxiras, Atomic and Electronic Structure of Solids, Cambridge University Press, 2003.

[22] http://silicon.physics.buffalo.edu/ dbkirby/hznocomplete. html, 2013. [Online; accessed 19-July-2013]. 


\section{List of Tables}

1 Crystal structure of wurtzite $\mathrm{ZnO}$, as obtained from [22] . . . 17

\section{List of Figures}

1 The $15 \times 15 \times 1$ supercell of wurtzite $\mathrm{ZnO}$ slab used as initial structure in our MD simulation. Left: Direct surface view from the direction $+z$; Right: Edge-on view. The $\left(\begin{array}{llll}0 & 0 & 0 & \overline{1}\end{array}\right)$ surface terminates with oxygen atoms while $\left(\begin{array}{llll}0 & 0 & 0 & 1\end{array}\right)$ Zn atoms. In these figures, the $\left(\begin{array}{llll}0 & 0 & 0 & \overline{1}\end{array}\right)$ surface is in the direction pointing along $+z$, while the $\left(\begin{array}{llll}0 & 0 & 0 & 1\end{array}\right)$ surface in the $-z$ direction. . . . . 18

2 A typical temperature vs. step profile in the simulation. . . . . 18

3 Ratio of $\mathrm{O}$ atoms sublimated (normalized to original number of $\mathrm{O}$ atoms on the surface) for $T=300$ to $1300 \mathrm{~K}$. . . . . . . 19

$4 \quad$ PL spectra for two wurtzite $\mathrm{ZnO}$ samples thermally treated at various temperatures. The intensity of the secondary peak in the spectrum first appears for $T=973 \mathrm{~K}$. At higher temperatures, the secondary peak records a higher intensity. . . . . . 20

$5 \quad$ Green luminescence $(512 \mathrm{~nm})$ intensity of the two $\mathrm{ZnO}$ samples at different temperatures. . . . . . . . . . . . . . 21

6 ESI-EFTEM images of white ZnO. Above: unannealed sample; Below: $N_{2}$ annealed $\left(700^{\circ} \mathrm{C}\right)$ sample. Red dot represents $\mathrm{Zn}$ atom while green dot $\mathrm{O}$ atom. Images reproduced from



7 Snapshots at various stages of the partial charge distribution in the slab when undergoing annealing at $T=300 \mathrm{~K}$. (a) At the beginning, the slab has only gone through energy minimization at $0.1 \mathrm{~K}$ but not any thermal treatment. (b) and (c) are snapshots during which the slab is being annealed at the temperature plateau $T$. (d) Slab at the end of thermal history as depicted by Fig. 2. The vertical axis is in units of $e$. The $z$-axis is in units of $\AA$. . . . . . . . . . . . . . . 22

8 Snapshots at various stages of the partial charge distribution in the slab when undergoing annealing at $T=1300 \mathrm{~K}$. . . . 23

9 Partial charge distributions at the end of an MD run for eight annealing temperatures ranging from $400 \mathrm{~K}$ to $1100 \mathrm{~K}$. . . . . 24 
10 Charge density $\rho(z)$ as a function of depth from the $\left(\begin{array}{llll}0 & 0 & 0 & \overline{1}\end{array}\right)$ surface, $z$, for annealing temperature $T=500 \mathrm{~K}, T=700 \mathrm{~K}$, $T=800 \mathrm{~K}$ and $T=1000 \mathrm{~K}$. The vertical axis is in units of $e / \AA^{3}$. The $z$-axis is in units of $\AA$. Note the qualitative change of the density profile (especially the region close to the $\left(\begin{array}{lll}0 & 0 & 0\end{array}\right.$ $\overline{1}$ ) end) when crossing from $T=700 \mathrm{~K}$ to $T=800 \mathrm{~K}$. . . . . 25

11 Average partial charge per sublimated atom as a function of annealing temperature. . . . . . . . . . . . . 26 
Table 1: Crystal structure of wurtzite $\mathrm{ZnO}$, as obtained from 22

\begin{tabular}{|c|c|c|c|c|}
\hline & & & & \\
\hline & & & & Unit Cell Parameters \\
\hline Fras & Col & atec of & atoms & $a=3.3500 \AA$ \\
\hline Atom & $x$ & $y$ & $z$ & $b=3.3500 \AA$ \\
\hline $\mathrm{O}$ & 0.3333 & 06667 & 03750 & $c=5.2200 \AA$ \\
\hline $\mathrm{Zn}$ & 0.0000 & 0.0001 & $\frac{0.0100}{0.0000}$ & $\alpha=90^{\circ}$ \\
\hline & & & & $\beta=90^{\circ}$ \\
\hline & & & & $\gamma=120^{\circ}$ \\
\hline
\end{tabular}



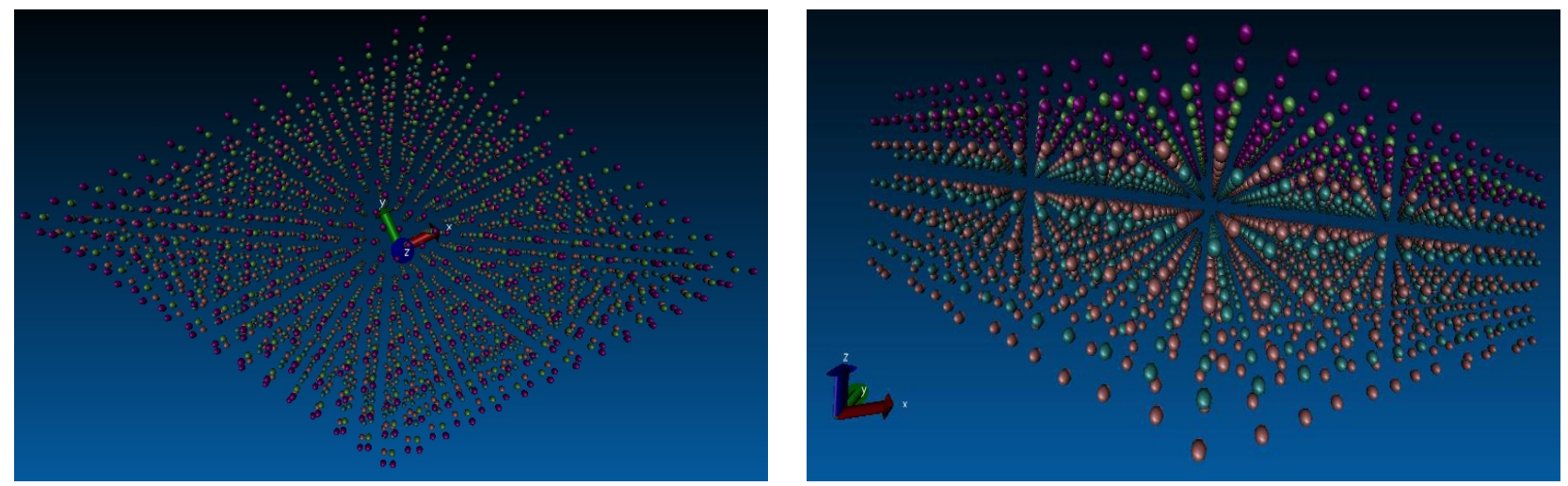

Figure 1: The $15 \times 15 \times 1$ supercell of wurtzite $\mathrm{ZnO}$ slab used as initial structure in our MD simulation. Left: Direct surface view from the direction $+z$; Right: Edge-on view. The $\left(\begin{array}{llll}0 & 0 & 0 & \overline{1}\end{array}\right)$ surface terminates with oxygen atoms while $\left(\begin{array}{llll}0 & 0 & 0 & 1\end{array}\right) \mathrm{Zn}$ atoms. In these figures, the $\left(\begin{array}{llll}0 & 0 & 0 & \overline{1}\end{array}\right)$ surface is in the direction pointing along $+z$, while the $\left(\begin{array}{llll}0 & 0 & 0 & 1\end{array}\right)$ surface in the $-z$ direction.



Figure 2: A typical temperature vs. step profile in the simulation. 




Figure 3: Ratio of $\mathrm{O}$ atoms sublimated (normalized to original number of $\mathrm{O}$ atoms on the surface) for $T=300$ to $1300 \mathrm{~K}$. 

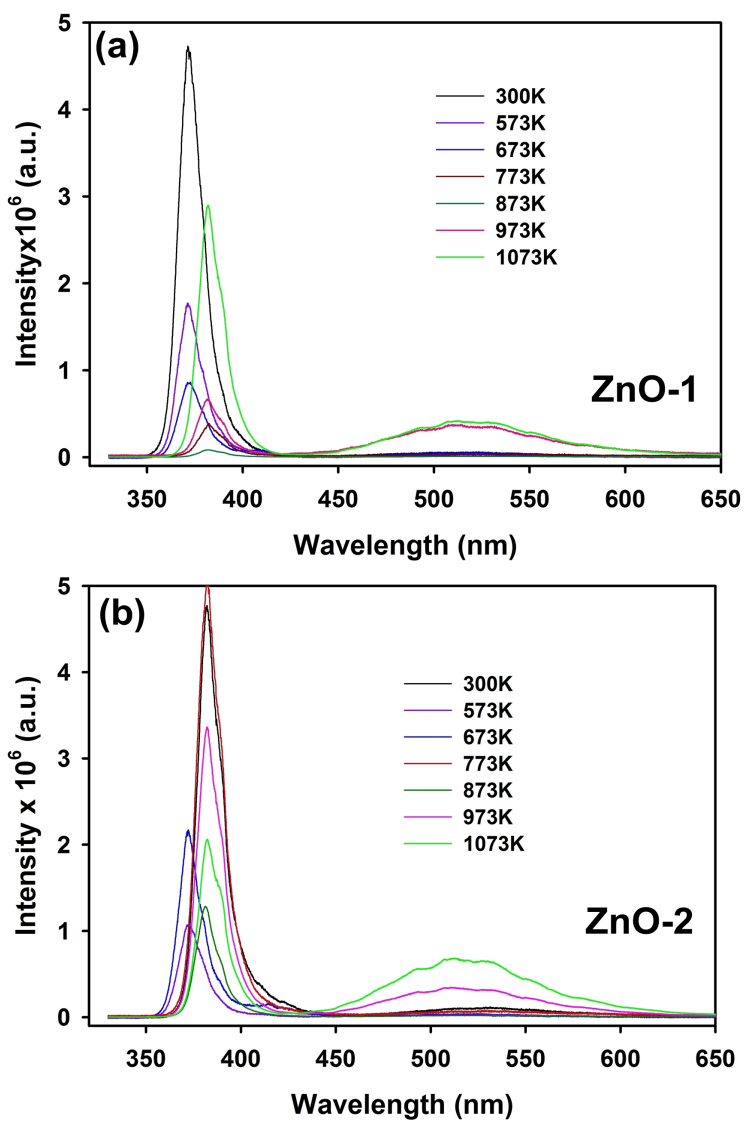

Figure 4: PL spectra for two wurtzite $\mathrm{ZnO}$ samples thermally treated at various temperatures. The intensity of the secondary peak in the spectrum first appears for $T=973 \mathrm{~K}$. At higher temperatures, the secondary peak records a higher intensity. 


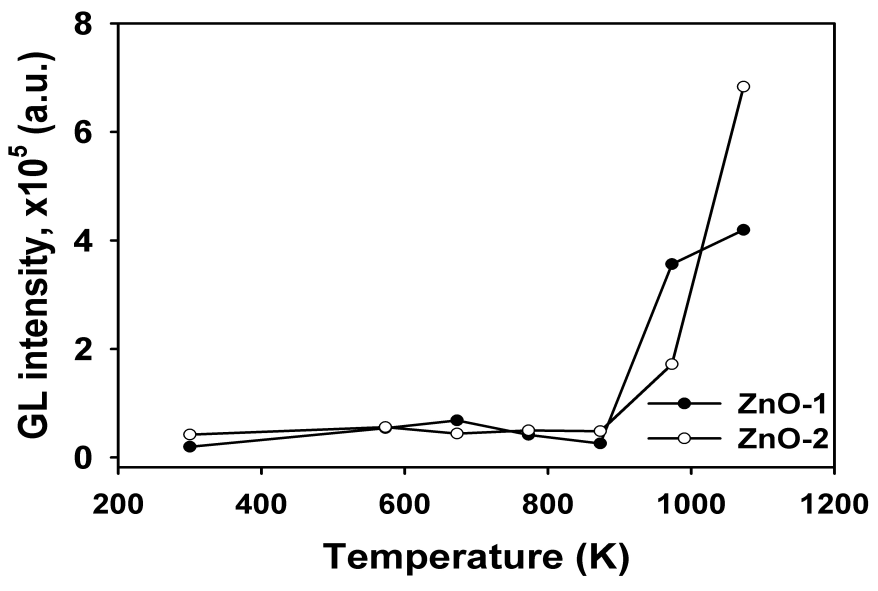

Figure 5: Green luminescence (512 nm) intensity of the two $\mathrm{ZnO}$ samples at different temperatures.

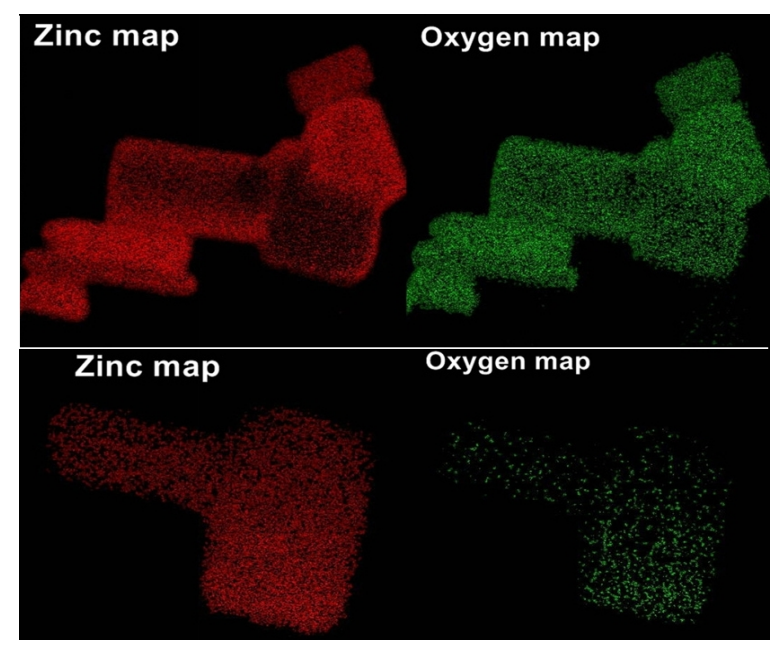

Figure 6: ESI-EFTEM images of white ZnO. Above: unannealed sample; Below: $N_{2}$ annealed $\left(700^{\circ} \mathrm{C}\right)$ sample. Red dot represents $\mathrm{Zn}$ atom while green dot $\mathrm{O}$ atom. Images reproduced from Ref. [20]. 

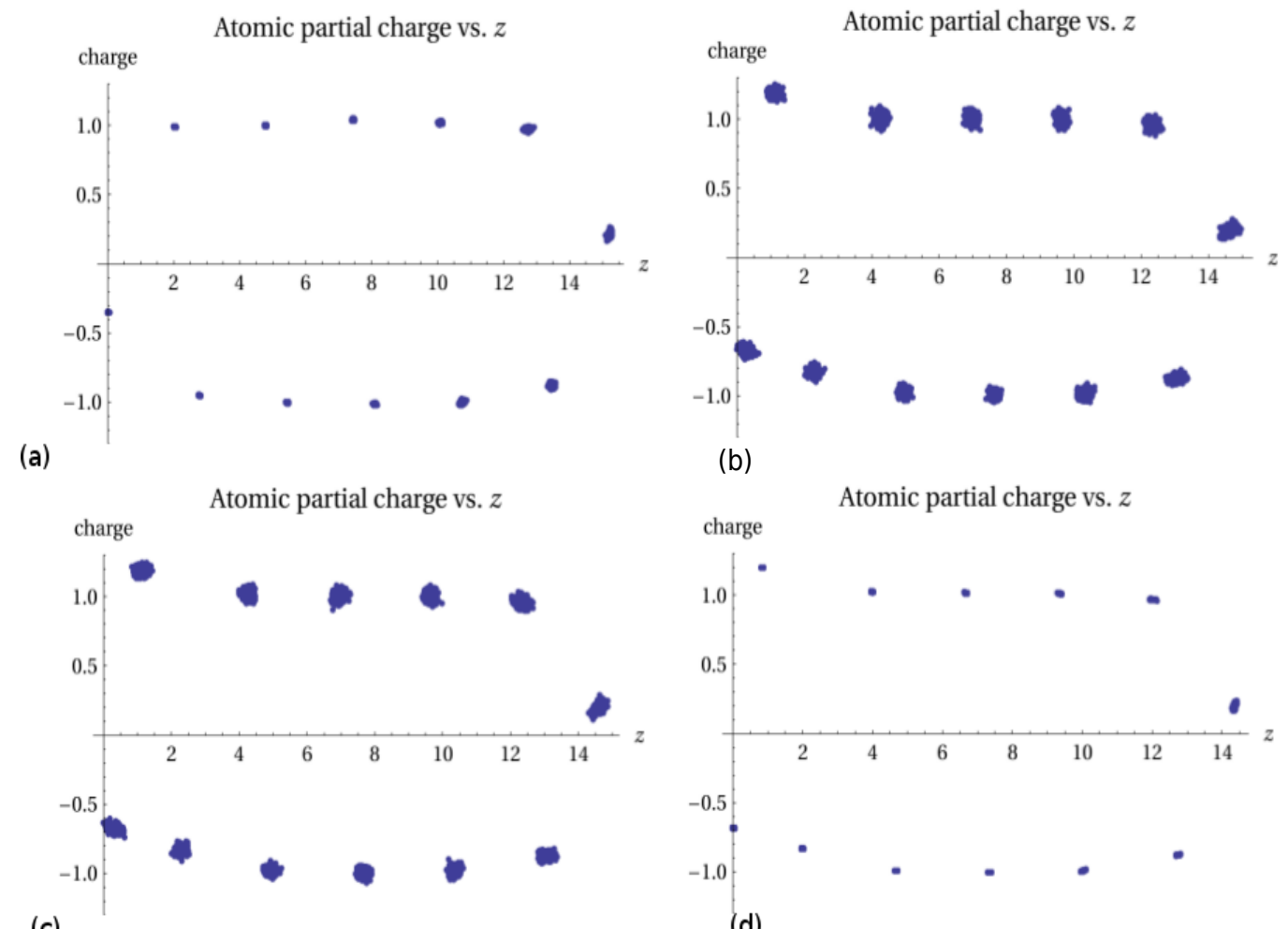

(b)

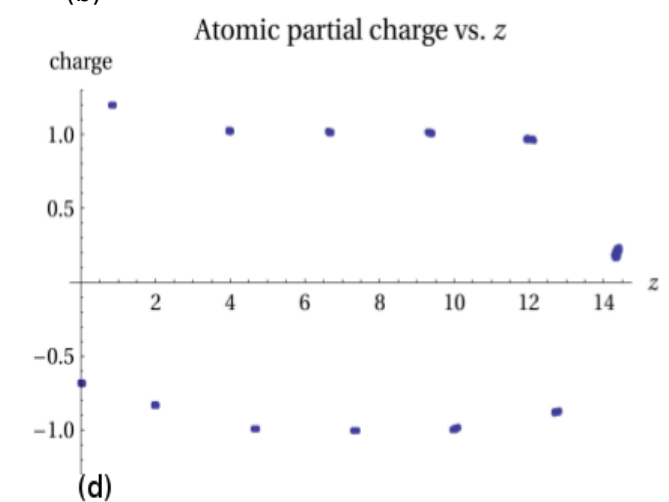

Figure 7: Snapshots at various stages of the partial charge distribution in the slab when undergoing annealing at $T=300 \mathrm{~K}$. (a) At the beginning, the slab has only gone through energy minimization at $0.1 \mathrm{~K}$ but not any thermal treatment. (b) and (c) are snapshots during which the slab is being annealed at the temperature plateau $T$. (d) Slab at the end of thermal history as depicted by Fig. 2. The vertical axis is in units of $e$. The $z$-axis is in units of $\AA$. 


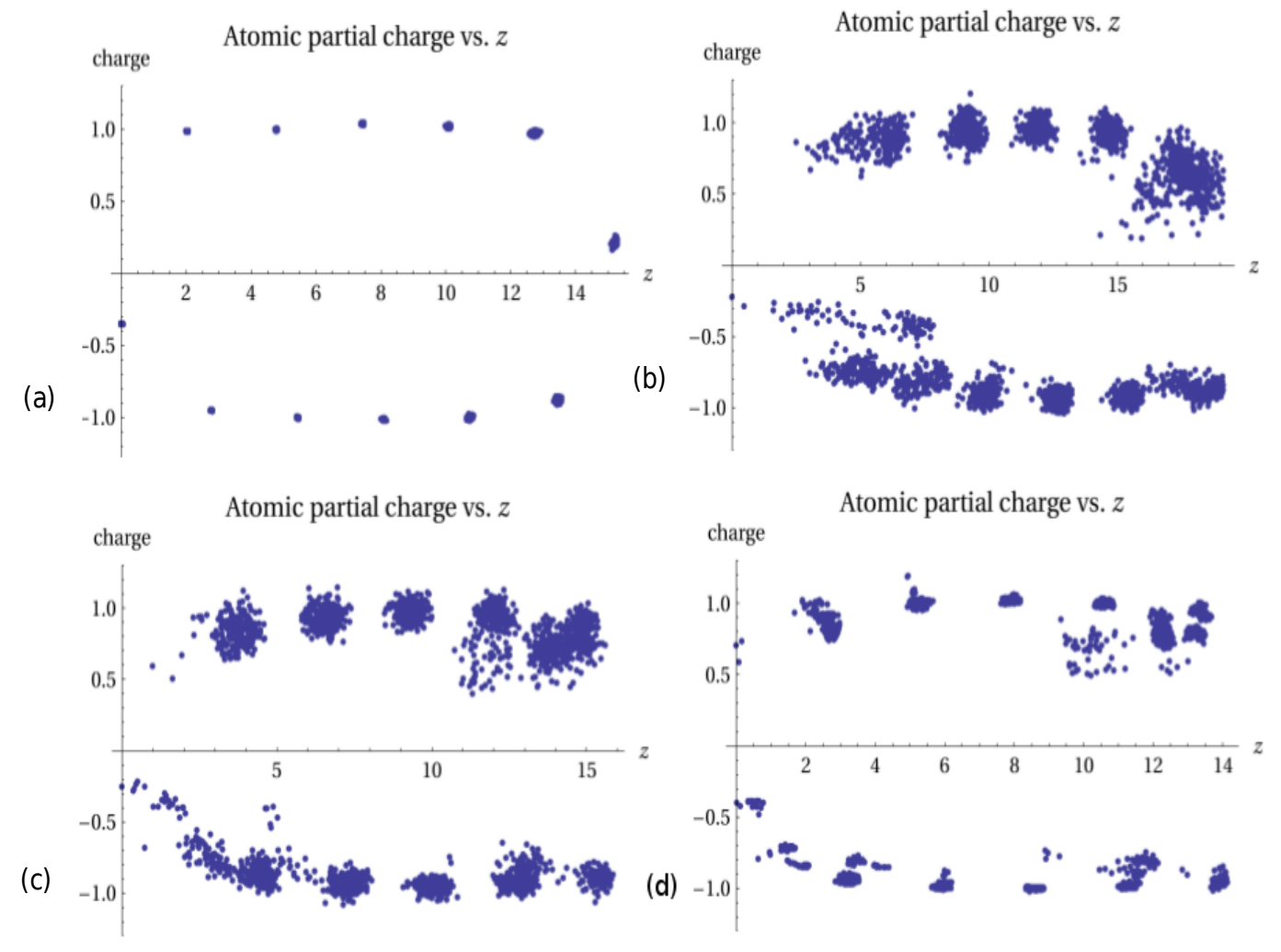

Figure 8: Snapshots at various stages of the partial charge distribution in the slab when undergoing annealing at $T=1300 \mathrm{~K}$. 

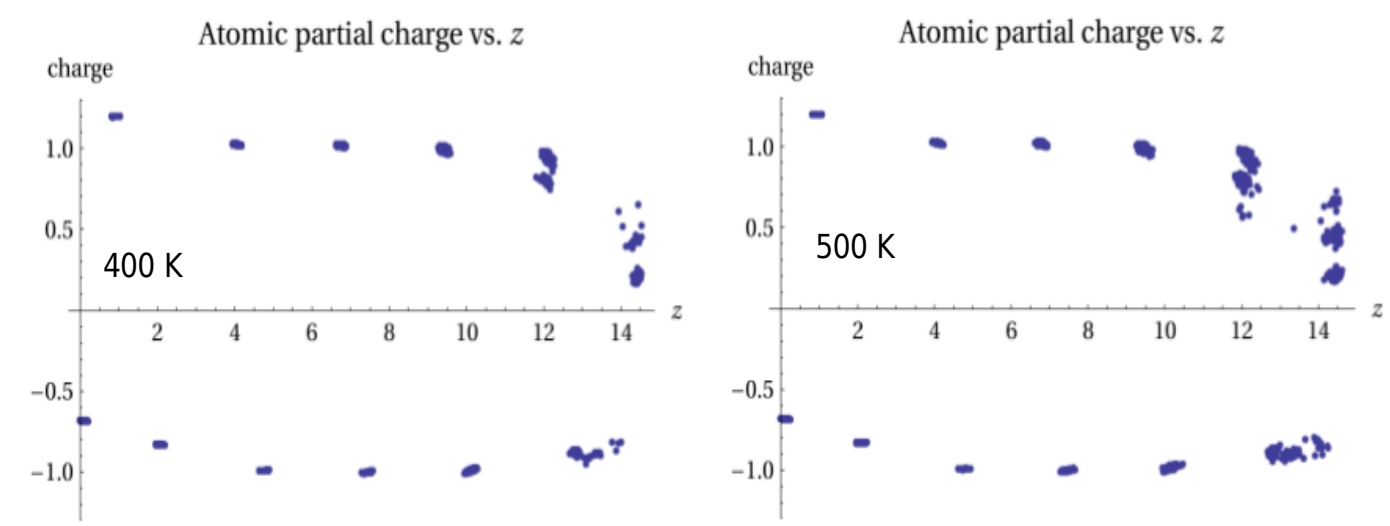

Atomic partial charge vs. $z$
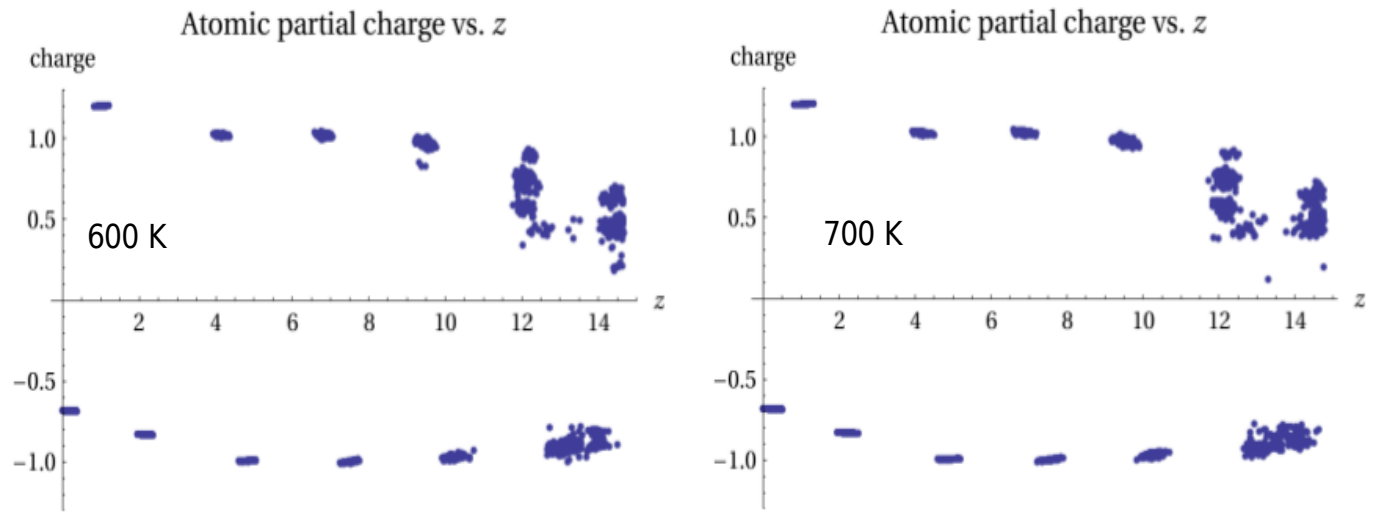

Atomic partial charge vs. $z$
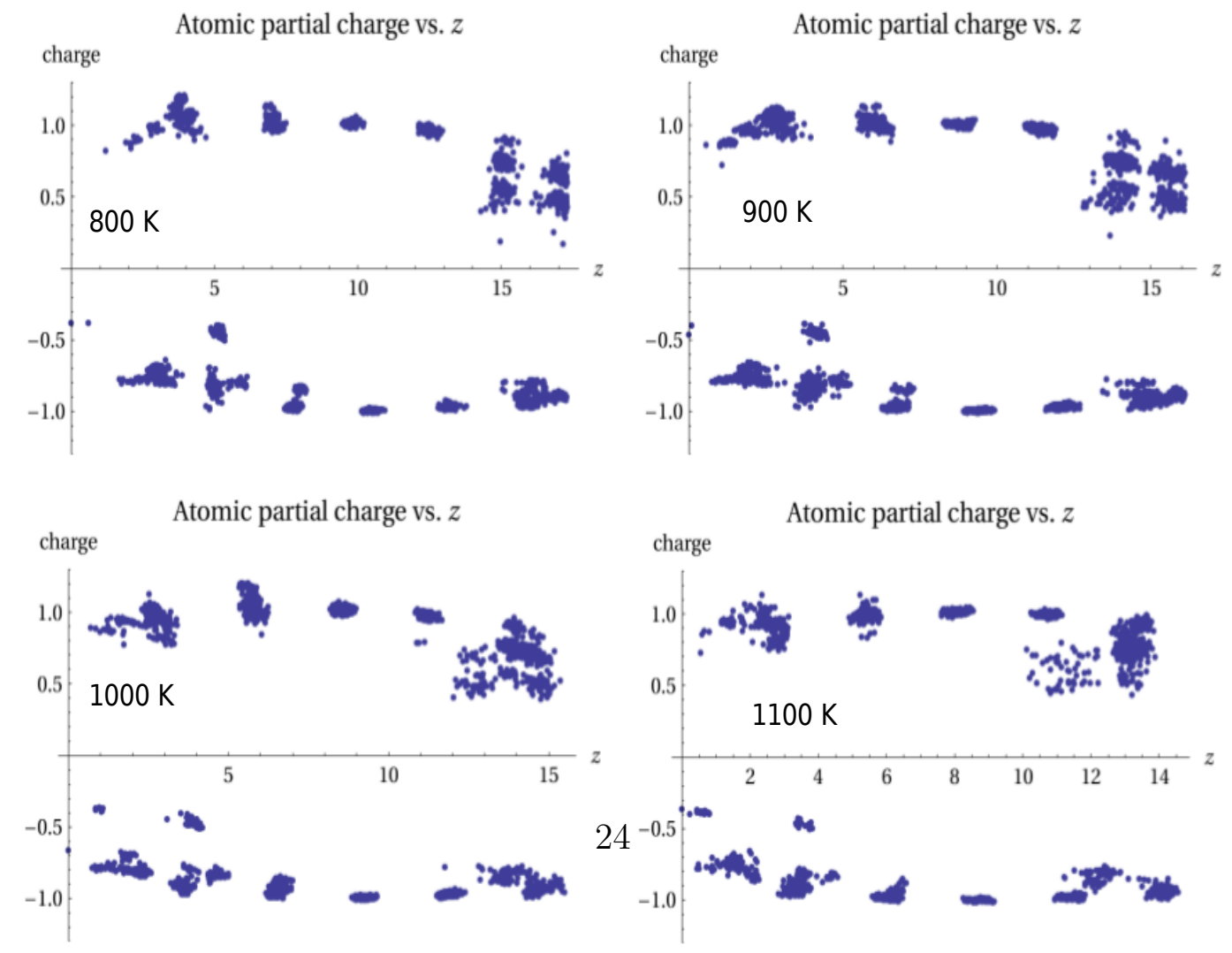

Figure 9: Partial charge distributions at the end of an MD run for eight annealing temperatures ranging from $400 \mathrm{~K}$ to $1100 \mathrm{~K}$. 

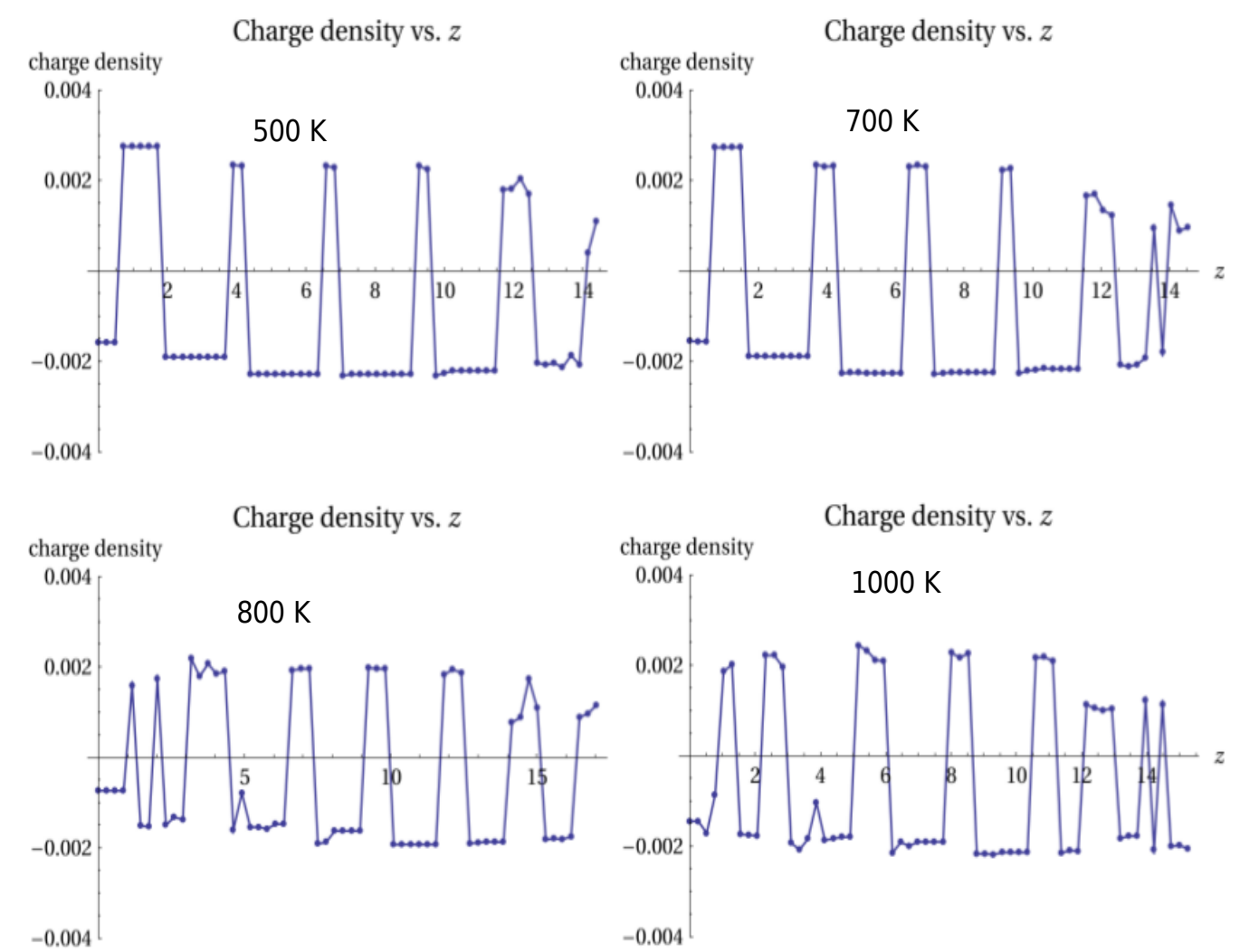

Figure 10: Charge density $\rho(z)$ as a function of depth from the $\left(\begin{array}{llll}0 & 0 & 0 & \overline{1}\end{array}\right)$ surface, $z$, for annealing temperature $T=500 \mathrm{~K}, T=700 \mathrm{~K}, T=800 \mathrm{~K}$ and $T=1000 \mathrm{~K}$. The vertical axis is in units of $e / \AA^{3}$. The $z$-axis is in units of $\AA$. Note the qualitative change of the density profile (especially the region close to the $\left(\begin{array}{llll}0 & 0 & 0 & \overline{1}\end{array}\right)$ end) when crossing from $T=700 \mathrm{~K}$ to $T=800 \mathrm{~K}$. 


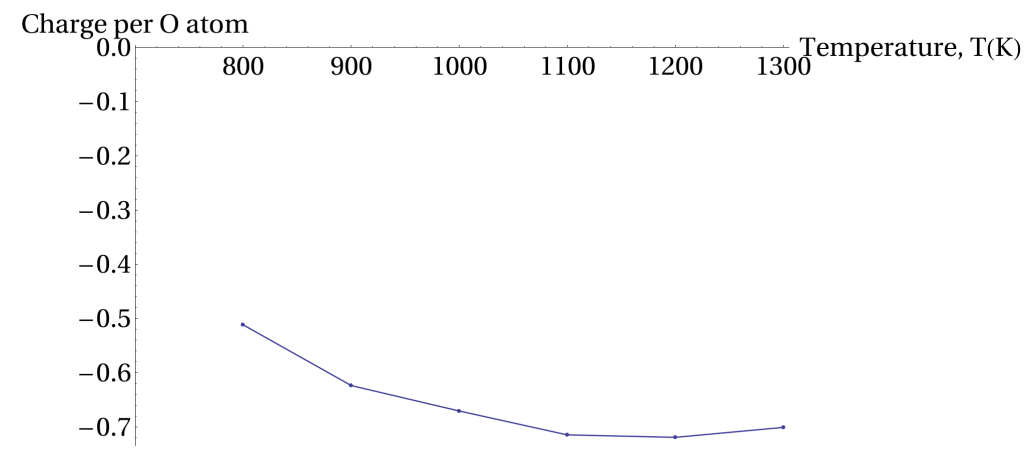

Figure 11: Average partial charge per sublimated atom as a function of annealing temperature. 